\title{
Educación en valores
}

\section{EDUCATION IN VALUES}

Miguel Ángel Polo Santillán

Universidad de Lima

mpolo@ulima.edu.pe

\section{RESUMEN}

El artículo presenta diferentes aspectos de la complejidad de la educación en valores. En primer lugar, la distinguimos y relacionamos con la educación moral y la educación ética. Seguidamente, vemos dos modelos de educación en valores, la apuesta por una educación en valores entendida como realización de la vida buena y el rol de los distintos actores sociales para la realización de esta propuesta.

PALABRAS CLAVE: valores, ética, educación en valores, educación ética, educación moral, modelos de educación en valores, vida buena

\section{ABSTRACT}

This paper presents different aspects of the complexity of education in values. First, we show that it should be distinguished from and related to moral and ethics education. Next, we analyze two models of education in values, the commitment to an education in values understood as the realization of the good life and the role of the different social actors in achieving this proposal.

KEYWORDS: values, ethics, education in values, ethics education, moral education, models of education in values, good life 


\section{INTRODUCCIÓN}

La educación en valores es una expresión sencilla, pero que involucra una complejidad de aspectos teóricos y prácticos que convoca a educadores, psicólogos y filósofos. $\mathrm{Si}$ bien es cierto que requerimos hacer distinciones desde la filosofía, también debemos hacer las articulaciones necesarias con otros elementos de esta realidad axiológica. Por eso, revisamos varios de estos aspectos, como esclarecer la diferencia entre educación en valores, educación ética y educación moral. También la reflexión sobre los modelos de educación en valores, sin olvidar su sentido teleológico y su articulación con la realidad social y cultural. Este modo complejo de asumir el tema nos ayudará a dejar de sobredimensionarla y tener una comprensión más realista del asunto.

\section{¿EDUCACIÓN MORAL? ¿EDUCACIÓN ÉTICA? ¿EDUCACIÓN EN VALORES?}

No siempre se tiene clara la distinción entre estos términos, por lo que en el mundo cotidiano pasan por sinónimos, en parte, debido a que en la misma filosofía no hay un consenso entre ética y moral. Por eso, la siguiente distinción presupone una definición de ética y de moral.

Por educación moral entenderemos la formación moral que se realiza dentro de un grupo con creencias determinadas; es lo que ocurre en un colegio religioso. Es una educación moral porque de lo que se trata es de transmitir y hacer que los alumnos interioricen y practiquen los valores de una determinada tradición religiosa o filosófica. En consecuencia, la educación moral busca reforzar las creencias, normas y valores morales de una comunidad.

Por educación ética entenderemos la formación crítica de una personalidad moral, es decir, que tendrá como elementos indispensables el pensamiento cuestionador, la mirada atenta y la búsqueda de buenas razones para actuar, teniendo como meta tanto la realización de la personalidad como la convivencia con otros grupos humanos con diferentes criterios morales'1.

La educación en valores suele entenderse como la capacidad de un sujeto de elegir sus valores para que orienten su existencia. Este tipo de educación debe despertar en el educando las capacidades racionales para que sepa discernir correctamente ${ }^{2}$.

1 Hoy la palabra ética ha alcanzado mayor prestigio, pues se habla de congresos de ética, cursos de ética, comités de ética, libros de ética, pero poco se habla de moral. Muchas razones pueden alegarse. En parte, esto se debe a que uno de los significados de ética tiene que ver con un pensamiento racional sobre los asuntos humanos que cuestionan las propias bases de las creencias morales tradicionales.

2 Por su parte, Payá (2000) considera que "la educación en valores y la educación moral son sinónimos" (p. 167). Por mi parte, sostengo que son diferentes aspectos de la educación. La diferencia está en lo que cada intelectual quiera darle a la moral, la ética y los valores. Pero lo importante es articularlos. 
No vamos a sostener la superioridad de uno de ellos, sino todo lo contrario: su mutua dependencia, aunque consideramos que el eje de todo esto debería ser una educación ética, ya que promueve un ejercicio libre y responsable de la vida de una persona. Y una educación moral sin compañía de una educación ética sería formar personas sin capacidad de cuestionar sus propias tradiciones, especialmente cuando limitan su capacidad de pensar por sí mismos y tomar sus propias decisiones. Y una educación en valores sin educación ética sería convertir la educación en un mercado donde se ofrezca a los compradores unos productos (valores) para que ellos elijan lo que deseen. La educación moral pone el acento en la tradición moral, la educación en valores lo pone en la libertad individual, mientras que ambas son afirmadas integralmente en una educación ética. Este puede ser un marco teórico para articular estos diferentes términos que suelen emplearse. Así, cuando hablemos de educación en valores, debemos pensarla integrada a la educación moral y a la educación ética ${ }^{3}$.

\section{PROPUESTAS DE EDUCACIÓN EN VALORES}

Este es el cómo de la educación en valores, especialmente en el área concerniente a los educadores y los psicólogos 4 . Ferreiros (1983, p. 220) desarrolla cinco tipos de enfoques para una educación en valores: inculcación, desarrollo moral, análisis, clarificación y aprendizaje para la acción. Desarrollemos brevemente cada enfoque.

a. Inculcación. Este enfoque busca infundir o internalizar determinados valores que son considerados deseables. Los motivos pueden ser inculcar valores universales o valores de alguna cultura. Este enfoque puede ser válido cuando la persona haya realizado su opción valorativa, pero su problema es que deja poco espacio para que se adhiera libremente a ellos.

b. Desarrollo moral. Este enfoque está sostenido en la teoría del desarrollo moral de Kohlberg (1992), quien lo divide en tres niveles, cada uno con dos pasos. En el nivel preconvencional, el sujeto tiene una perspectiva egocéntrica. Si busca lo bueno, es por la recompensa; si evita lo malo, es por el castigo. En el nivel convencional, el sujeto se orienta por reglas de orden social; por eso se circunscribe a criterios grupales, como familia, grupo o nación. En el tercer nivel, posconvencional, el sujeto se orienta por principios universales, como los derechos humanos. El problema de este enfoque es que prevalece lo cognitivo, subordinando los sentimientos y las actitudes; además, se centra en los valores morales y no percibe la importancia de los demás valores en el desarrollo moral. Tampoco estos niveles tienen que anularse.

3 Otros aspectos filosóficos sobre la educación en valores los he tratado en los artículos "El significado de educar en valores" (2005) y “Los problemas en los discursos de los valores" (2008).

4 Otros trabajos sobre cómo educar en valores: Paniego (1999) y Carreras et al. (1996). 
c. Análisis. Utiliza el procedimiento lógico y de investigación científica para aplicarlo a los valores, especialmente del campo social. Busca que la validez de los valores esté acompañada con verificación a través de los hechos. La crítica a este enfoque es su peso en la racionalidad científica y la creencia de que hasta en los valores es posible una investigación objetiva y controlada. Y de ser posible, dejaría la educación bajo el control de los sujetos racionales, los maestros-científicos.

d. Clarificación. Su objetivo es ayudar al estudiante a reconocer aquello que es un valor en su vida, para poder fortalecerlo o cambiarlo. En este enfoque es importante elegir libremente los valores, los cuales, una vez asumidos, serán apreciados; con ello se puede garantizar el actuar de acuerdo con sus propios valores. Al interesarse en que el individuo se haga consciente de sus propios valores, puede caer en un enfoque individualista, dejando de lado asuntos como conflictos de valores o la necesidad de reconocer valores cívicos.

e. Aprendizaje para la acción. El objetivo de este enfoque es relacionar los valores con las acciones, es decir, actuar según los valores. Hay mayor peso en las acciones por realizar que en los valores mismos.

En el texto de Payá (2000, p. 168) se sintetizan las propuestas de educación en valores en cuatro. Vamos a presentarlas y luego detenernos en una propuesta integral.

a. Educación en valores entendida como clarificación. La educación en valores ayuda en la reflexión de los valores, no en su transmisión. La clarificación de los valores busca que los estudiantes tengan una visión crítica de sus vidas. De ahí que los tres pasos necesarios deben ser conocimiento de sí, clarificación de la propia postura personal y construcción del yo. La crítica que hace Payá (2000) es que tiene un enfoque "individual y subjetivo de la educación en valores" (p. 172), así como que no trabaja el tema de los conflictos de valor que aparecen en la vida en comunidad.

b. Educación en valores entendida como formación del carácter. De tradición aristotélica, este enfoque busca la formación de hábitos virtuosos, base de una personalidad moral, además de que ello dependerá del proyecto de "vida buena" que se tenga. La crítica de Payá (2000) es que "soslaya el cuestionamiento" (p. 175). Además, da importancia a los modelos de vida virtuosa, pero no tanto al "proceso de elaboración personal" (p. 176) desde su propia subjetividad y experiencia.

c. Educación en valores entendida como proyecto de vida. Sobre la base del pensamiento aristotélico, se pone acento en el proyecto ideal de vida, para lo cual hay que ejercer la autonomía, la reflexión y la deliberación individual, pero dentro del contexto comunitario. Considera Payá (2000) que este modelo "suele quedar limitado a la esfera de la iniciativa personal" (p. 184). 
d. Educación en valores entendida como construcción de la personalidad moral. Esta es la propuesta que sostiene Payá (2000), porque asume las cosas buenas y supera los problemas de las anteriores propuestas, por lo que la considera un "modelo completo". Tiene sus fuentes en la filosofía kantiana y neokantiana, así como en la tradición cognitivo-evolutiva. En esta propuesta de educación en valores, se tienen en cuenta cuatro elementos centrales: (i) la racionalidad crítica que supera el subjetivismo; (ii) la libertad y la autonomía, sustento del respeto a la persona; (iii) el diálogo, instrumento mediante el cual se ejerce la crítica, la comprensión y la optimización, a partir del cual se puede llegar a consensos; y (iv) la construcción de su propia dimensión valorativo-moral tanto en lo privado como en lo público. Así, autonomía y razón dialógica se convierten en principios esenciales con los cuales el estudiante puede construir significativamente sus valores.

Este enfoque "permite abordar, de forma integrada, respetuosa con la individualidad y con un contenido susceptible de universalización, el desarrollo de la dimensión valorativo-moral de la persona" (Payá, 2000, p. 190). La tabla 1 resume lo expresado por Payá y agrega algunas ideas a estas propuestas.

Tabla 1

Resumen de las cuatro propuestas de educación en valores sintetizadas por Payá (2000)

\begin{tabular}{|c|c|c|}
\hline $\begin{array}{l}\text { Propuesta de } \\
\text { educación en valores }\end{array}$ & Tesis centrales & Crítica \\
\hline Clarificación & $\begin{array}{l}\text { Posibilita el autoconocimiento y el } \\
\text { conocimiento entre los miembros del } \\
\text { grupo. } \\
\text { Estimula el análisis y la reflexión como } \\
\text { procedimientos de valoración moral. } \\
\text { Procura unir pensamiento, afecto y } \\
\text { comportamiento. }\end{array}$ & $\begin{array}{l}\text { No garantiza el paso del juicio a la } \\
\text { acción. } \\
\text { El relativismo es una consecuencia de } \\
\text { sus propuestas. }\end{array}$ \\
\hline Formación del carácter & $\begin{array}{l}\text { Permite el desarrollo de hábitos } \\
\text { virtuosos. } \\
\text { Da importancia a los modelos, a los } \\
\text { cuales hay que emular. }\end{array}$ & $\begin{array}{l}\text { Su actuar se circunscribe a principios } \\
\text { y valores dentro de tradiciones que } \\
\text { tienen una forma de entender la "vida } \\
\text { buena". }\end{array}$ \\
\hline Proyecto de vida & $\begin{array}{l}\text { La dimensión valorativo-moral está } \\
\text { relacionada con la vida de la persona } \\
\text { como totalidad. } \\
\text { El proyecto ideal equilibra lo cognitivo } \\
\text { y lo comportamental, lo privado y lo } \\
\text { público. }\end{array}$ & $\begin{array}{l}\text { No proporciona guía para construir y } \\
\text { recorrer ese proyecto de vida. } \\
\text { Falta de pasos intermedios. }\end{array}$ \\
\hline $\begin{array}{l}\text { Construcción de la } \\
\text { personalidad moral }\end{array}$ & $\begin{array}{l}\text { Desde los principios de autonomía } \\
\text { y razón dialógica, la persona puede } \\
\text { realizar una construcción significativa } \\
\text { de su ámbito valorativo-moral. }\end{array}$ & $\begin{array}{l}\text { Se pone énfasis en el carácter racional, } \\
\text { subordinándose los sentimientos. }\end{array}$ \\
\hline
\end{tabular}


La educación en valores no debería cerrar la búsqueda de valores ni la búsqueda de la vida buena. Debe ayudarnos a prender la chispa de la búsqueda, porque en esa misma búsqueda está la realización.

\section{EDUCACIÓN EN VALORES ENTENDIDA COMO REALIZACIÓN DE LA VIDA BUENA}

La búsqueda del "cómo" no debe hacernos perder el sentido, la finalidad de la educación. Queremos entender que la tarea educativa es una actividad por la cual se procura ofrecer los elementos necesarios para que las personas vayan realizando su vida buena. Esto no quiere excluir las propuestas anteriormente señaladas, sino abarcarlas ${ }^{5}$. Por eso debemos tener en cuenta, a la hora de la realización del proyecto de una educación en valores, los grandes aspectos que conforman la personalidad. En esta parte, se revisarán las necesidades humanas en su conexión con la educación en valores, así como las dimensiones de la personalidad que deben ser trabajadas.

Por lo anterior, una buena propuesta de educación en valores debe estar basada en un conocimiento del desarrollo de la personalidad. No es casualidad que muchas de las propuestas de educación en valores hayan venido de la psicología. ¿Qué factores psicológicos hay que tomar en cuenta para la educación en valores? En principio, casi todos. Por eso, la educación en valores que desarrolle el profesor debe estar acompañada por los psicólogos educacionales que trabajan en el centro educativo.

Los psicólogos hablan de distintos tipos de desarrollo: el desarrollo psicosexual, el desarrollo psicosocial, el desarrollo emocional, el desarrollo moral, etcétera. Si bien los educadores necesitamos tener en cuenta estos tipos de desarrollo, por principio la educación promueve un desarrollo integral de toda la personalidad. Sin embargo, el educador debe optar por un modelo de desarrollo de la personalidad que le pueda servir de orientación para su trabajo. Nosotros presentaremos la teoría de las necesidades de Abraham Maslow y señalaremos que su propuesta requiere tener en cuenta los estratos de la personalidad.

Maslow (1991) propuso una jerarquía de las necesidades, las cuales se ordenan en forma piramidal. En la base de la pirámide están las necesidades fisiológicas, luego la necesidad de seguridad, después las de amor y pertenencia, luego la de la estima, la de autorrealización y, como culminación, está la necesidad de trascendencia. Hay una continuidad entre los valores de base y los espirituales. Indica el autor que las necesidades básicas son necesarias para las demás. Donde una necesidad está satisfecha, la siguiente presiona para su satisfacción. Maslow (1991) entiende que estas

5 La educación en valores como construcción de la personalidad moral, trabajada por Payá (2000), no es significativamente diferente de lo que nosotros entendemos por realización de la vida buena. Solo el enfoque antropoético que le queremos dar pone el acento en otros elementos. 
son las raíces biológicas de la vida valorativa. Reflexionemos, entonces, sobre lo que nos dice:

1. Los individuos que se autorrealizan (más maduros, más plenamente humanos), por definición ya adecuadamente gratificados en cuanto a sus necesidades básicas, se encuentran ahora motivados en otros sentidos superiores, a los que llamamos metamotivación. [...]

8. La vida valorativa (espiritual, religiosa, filosófica, axiológica, etcétera) es un aspecto de la biología humana y pertenece al mismo continuo que la vida animal e "inferior" (en vez de pertenecer a campos separados, dicotomizados o recíprocamente excluyentes). [...]

10. Dado que la vida espiritual es instintiva, todas las técnicas de la "biología subjetiva" se aplican a su educación.

Dado que la vida espiritual es parte del ser real, que es instintivo, es en principio susceptible de introspección. Tiene "voces impulsivas" o "señales interiores" que, aun siendo más débiles que las necesidades básicas, se llegan a "oír" y entran, por consiguiente, bajo el rubro de la "biología subjetiva".

En principio, por ende, todos los principios y ejercicios que nos ayudan a cultivar (o enseñar) nuestra percepción sensorial, nuestra conciencia corporal, nuestra sensibilidad a las señales provenientes de nuestro interior (emitidas por nuestras necesidades, capacidades, constitución, temperamento, cuerpo, etcétera), todo esto es válido también, aunque con menos fuerza, para nuestras metanecesidades interiores, es decir, para la educación de nuestra avidez de belleza, justicia, verdad, perfección, etcétera. (pp. 182-191)

Estas ideas de Maslow (1991) deben servirnos para apreciar la dinámica entre necesidades y valores, además de reconocer que el trabajo de los valores no es un asunto de ideas desconectadas de las necesidades de las personas, sino de saber estar atentos a las "señales interiores". Como dice Díaz (2001), así como hay personas ciegas a los valores, los hay también quienes son ciegos a sus necesidades. Por eso aboga por la educación de las necesidades a la par de educar en valores. Porque si queremos recorrer todo el planeta, viajar a la Luna, tener posesiones en muchos países, dominar a muchas personas, etcétera, entonces, no sabemos lo que necesitamos. Al no tener educadas nuestras necesidades, terminan imponiéndose las necesidades superficiales o aberrantes. "Las personas con una jerarquía de necesidades muy desenfocada reconocen una escala de valores muy desenfocada" (Díaz, 2001, p. 133).

Esta teoría de las necesidades humanas es una perspectiva diacrónica que necesita ser completada con una perspectiva sincrónica en la que podamos considerar los objetivos por trabajar: los conocimientos, las habilidades y las actitudes. Veamos la tabla 2, elaborada por Diego Gracia (2000) para sus trabajos de bioética. 
Tabla 2

Perspectiva sincrónica de la educación en valores

\begin{tabular}{llll}
\hline $\begin{array}{c}\text { Estratos } \\
\text { de la personalidad }\end{array}$ & $\begin{array}{c}\text { Objetivos } \\
\text { educacionales }\end{array}$ & $\begin{array}{c}\text { Metodologías } \\
\text { bioéticas }\end{array}$ & $\begin{array}{c}\text { Duración } \\
\text { temporal }\end{array}$ \\
\hline Actos & Conocimientos & $\begin{array}{l}\text { Principios } \\
\text { Consecuencias }\end{array}$ & $\begin{array}{l}\text { Corta } \\
\text { duración }\end{array}$ \\
Hábitos & Habilidades & $\begin{array}{l}\text { Virtudes } \\
\text { Vicios }\end{array}$ & Media \\
& Actitudes & Hechos \\
Carácter & Valores & Larga \\
& & duración \\
\hline
\end{tabular}

Fuente: Gracia (2000)

Recordemos una vez más a Aristóteles, quien expresaba que debemos tener en cuenta tres verbos: saber, elegir y actuar. Aunque la educación en valores no es formación en valores, es decir, solo transmitir conocimientos sobre los valores, el componente cognitivo es inevitable. Aquí es donde debemos fomentar la racionalidad crítica y dialógica a través del conocimiento de casos, reflexión en pequeños grupos, debates e investigaciones sobre valores, estudio de situaciones de conflictos de valores, etcétera. Sin este nivel cognitivo, no habría elecciones correctas. Conocer los valores y su sentido nos lleva a ver su ligazón con las normas, las cuales orientan nuestras acciones.

Este nivel cognitivo va generando habilidades en el diálogo, en la forma de lograr consensos, en la mirada crítica, en la identificación de conflictos de valor y posibles procedimientos para solucionarlos. Pasar a las habilidades no es un asunto de pocas sesiones, sino de acciones continuas y planificadas. Por eso la educación en valores no puede ser dejada a la voluntad de los profesores, sino que tiene que ser asumida también por otros profesionales y la comunidad familiar. Desde el plano de la ética, se puede ver en este nivel la importancia que le daba Aristóteles a los hábitos, es decir, a las acciones que se repiten en el tiempo y van formando carácter. Sin hábitos, no hay posibilidad de virtudes, de excelencias.

En tercer lugar, están las actitudes, las cuales se vuelven características de nuestra personalidad, que se forma con el tiempo. Las actitudes son disposiciones estables que son expresiones de una personalidad; están conformadas por el componente afectivo. Con ello, las actitudes expresan los valores que una persona ha asumido. Sin ellas, los valores no pasan de ser ideales bonitos o instrumentos para resolver conflictos; sin ellas, no se llega a tener un significado vital. En esta relación entre valores y actitudes, Payá (2000, p. 153) hace estas observaciones: 
a. Los valores engloban a las actitudes, porque las dirigen y les dan su sentido.

b. Un único valor puede abarcar diversas actitudes. Por ejemplo, la amistad abarca actitudes como la confianza, la sinceridad, la cooperación, etcétera.

c. Una misma actitud puede referirse a diferentes valores. Por ejemplo, los valores de la amistad, la solidaridad y el trabajo requieren frecuentemente de la actitud de la cooperación.

Puesto en términos éticos, para lograr el desarrollo de la personalidad ética se requiere de valores, normas y virtudes. Los valores como ideales dan sentido a la existencia y a las acciones, pero se expresan en normas cuyo carácter prescriptivo nos exige actuar de una manera. Todo ello va creando actitudes que moldean la personalidad.

Como puede verse, esto está ligado a los contenidos educativos frecuentemente mencionados en educación: los contenidos conceptuales, procedimentales y actitudinales.

Los contenidos conceptuales abarcan los conocimientos de los hechos, de los conceptos y los principios. Aquí se espera lograr aprendizajes memorísticos y de comprensión. Es el ámbito del saber. Los contenidos procedimentales se refieren al uso de distintas acciones y estrategias para realizar los objetivos propuestos; en este nivel se integran el aprendizaje en conocimientos y la práctica de habilidades. Es el ámbito del saber hacer. Los contenidos actitudinales se refieren a las actitudes, las que expresan vivencia de valores y normas. Es una predisposición a actuar de una forma racional, autónoma y dialógicamente. Es el ámbito del ser, de la formación de la personalidad ética. Sin ella, los valores pueden quedarse como contenidos teóricos sin trascendencia vital. No se trata de apostar a una idea fuerte de ser, sino de la comprensión de nuestra condición terrestre y cósmica, de nuestra individualidad y humanidad, de la unidad y diversidad que somos. Como señala Morin (1999):

Desde ahora, una ética propiamente humana, es decir, una antropoética debe considerarse como una ética del bucle de los tres términos, individuo $\leftrightarrow$ sociedad $\leftrightarrow$ especie, de donde surgen nuestra conciencia y nuestro espíritu propiamente humano. Esa es la base para enseñar la ética venidera. (p. 59)

Finalmente, debemos tener en cuenta otros elementos. En primer lugar, no confundamos los valores que puede asumir una persona en alguna etapa de su vida, con el valor supremo de realizarse como persona. Cuando se es adolescente, adulto, anciano, las personas pueden ir cambiando de valores o dándoles nuevos sentidos a los que tenían o dándoles pesos diferentes en cada etapa. Pero todos estos cambios deben estar en coherencia con la finalidad de la existencia, lo cual siempre implica valores personales y públicos. 


\section{PASOS PARA LA EDUCACIÓN EN VALORES}

La educación en valores no es una asignatura que se cubra totalmente en un tiempo escolar, sino que, empezando en el colegio (o en el hogar si es posible), debería continuar toda la vida. La educación ética, de la que los valores forman parte, es un ejercicio vital, permanente, pero también comunitario. El eje de los pasos que vamos a presentar debe ser la actitud crítica y el diálogo, los cuales pueden garantizar su realización. Tendremos en cuenta lo trabajado por Payá (2000).

- Clarificación. Las personas, sobre todo los adolescentes, no siempre tienen claro el sentido de los valores ni los valores de sus propias vidas y tradiciones. Este marco confuso y contradictorio de los valores que han asimilado debe ser sometido a una clarificación. Muchos jóvenes son cristianos de tradición, pero no conocen los grandes valores de su religiosidad. Los maestros no deberían empezar inculcando valores sin antes trabajar el terreno, es decir, ver el mundo conflictivo de valores que ya posee un alumno. En este proceso de autoconocimiento, los estudiantes también pueden descubrir la jerarquía de sus valores y las fuentes de donde derivan sus valores. Esto también les puede permitir conocer las dificultades que existen en la realización de sus valores y los pasos que deben seguir los maestros.

- Autoposesión de valores. Viendo el mundo de sus valores, su sentido, los conflictos y jerarquías que tiene, buscará la mejor manera de construir su propio mundo de valores, dejando de lado los que son obstáculos a su desarrollo personal y a la finalidad de su vida, asumiendo con claridad los valores que orientarán su existencia. Con ello, tendrá que reconocer las condiciones que requieren el cumplimiento de sus valores (trabajo de las actitudes, de los sentimientos, de la racionalidad, una comunidad de acompañamiento, etcétera), así como las dificultades posibles. Descubrir sus valores sin dar el paso de la incorporación dentro de un proyecto de vida hace de los valores simples entidades metafísicas.

- Coherencia entre valores y vida. El alumno debería estar capacitado para observar críticamente las incoherencias entre los valores vividos y los valores proclamados. La comunidad de apoyo (familia, amigos, profesor) muchas veces nos puede ayudar en este aspecto, porque uno mismo a menudo no ve sus propios errores. Al no ser entes cerrados ni acabados, sino abiertos al mundo y a la vida, la participación de los otros en su propio desarrollo moral se hace indispensable.

- Reforzamiento de los valores. Ello requiere de un estilo de vida adecuado que pueda estimular y reforzar los valores apropiados. Y, por la propia naturaleza 
de los valores, asumir algunos de ellos implicará apreciar o rechazar ciertos modos de vivir y actuar. Se necesitan espacios de afirmación de nuestros valores, como ver determinadas películas o periódicos, asistir a ciertas charlas o exposiciones artísticas, crear espacios de diálogo y reflexión, encuentros con amigos, etcétera. Razón tenía el filósofo chino Mencio (2012) cuando sostenía: "Aprender de otro a hacer el bien es ayudar a otros a hacer el bien haciéndolo junto a ellos" (p. 75). Hacer el bien junto a otros sigue siendo una enseñanza pedagógica para nuestro tiempo.

Finalmente, no creo que debamos rechazar lo bueno de las distintas propuestas de educación en valores. Sus problemas generalmente han sido sus visiones parciales, pero ello se puede superar si tenemos en cuenta una gama de indicadores, como el respeto a la individualidad y a la comunidad, la atención al carácter, el trabajo de la razón dialógica, entre otros.

\section{DE LOS PROFESORES A LA SOCIEDAD}

En el colegio existen distintos niveles de responsabilidades de la educación en valores: los maestros, las autoridades, los padres de familia y la comunidad. El educador adquiere importancia porque es quien dirige la búsqueda del significado de la existencia del educando, así como la suya propia. Y en esa búsqueda es que ambos van dando los pasos necesarios para su autorrealización. Como sostiene Díaz (2001):

En el caso del maestro, a pesar de su menor prestigio social, y de su importancia decreciente, su vocación es la de vivir ayudando a los demás a realizar su proyecto existencial, pese a la dificultad de la tarea y la humana fragilidad. (p. 166)

Las propuestas de educación en valores las hacen los profesores, los padres de familia y el Estado (recuérdese que el Ministerio de Educación tiene un modelo educativo que encierra valores, pero que pocas veces es conocido por los padres). Claro que la elección y desarrollo debería hacerse dialógicamente, involucrando a toda la comunidad escolar. Los maestros, los padres y los hijos deben participar en esta educación. De lo contrario, los jóvenes no tendrán la comunidad y el ejemplo necesarios para hacer suyos los valores. Pero es el profesor quien tiene un mayor peso de responsabilidad, por lo que es necesario, al poner en ejecución la educación en valores, estar preparado y con voluntad de llevarla a cabo. Y también vale recordar que los diferentes objetivos y métodos tendrán que adaptarse a la edad de los alumnos.

Es importante, además, reconocer que los otros factores que intervienen en la relación profesor-alumno no son neutrales axiológicamente. La estructura del colegio, la organización de las actividades, la metodología didáctica, la comunidad de docentes y padres contienen valores, sea de manera implícita o explícita. Ellos se convierten 
en medios por los cuales los estudiantes van asimilando valores. Por eso: "La toma de decisiones acerca de cualquier elemento que integra el 'estilo' pedagógico de una determinada escuela implica una opción de los valores. Tal opción puede ser realizada consciente o inconscientemente" (Ferreiros, 1983, p. 206).

Por último, debemos ser conscientes de que la tarea de la educación en valores no queda circunscrita a la educación escolar, de ahí la necesidad de convocar a la familia, la sociedad civil (empresas, organizaciones no gubernamentales, universidades, iglesias, etcétera) y las instituciones políticas, campos donde se ponen en juego los valores. Se debe reconocer los tipos de valores que se pueden defender en cada ámbito y la riqueza que adquiere la dinámica social con ellos o la degradación sin ellos. La formación en valores debe ser realizada en las aulas escolares si vemos un mundo que las niega, para lo cual se necesitará de nuevos instrumentos y estrategias para hacer frente a esa realidad.

\section{REFLEXIONES FINALES}

Tenemos que reiterar que es necesario articular la educación en valores con la educación ética y la educación moral; de ese modo, la relación entre la teoría y la práctica, la crítica racional con las acciones y la formación de hábitos pueden tener más consistencia. Su fragmentación tiende a generar fracasos o dogmatismos. Fracasos de una enseñanza intelectualista de los valores o dogmatismo moral que no cuestiona nada de su propia tradición.

La realización de los valores requiere tener en cuenta tres aspectos:

a. El para qué, el sentido de la enseñanza en valores: ¿qué tipo de persona o ciudadanos queremos formar?, y desde ahí ver los procedimientos.

b. Dejar de creer que la educación en valores se reduce a la educación en valores éticos, desvalorizando los demás valores que hacen una vida digna y vivible. Por ejemplo, los valores intelectuales, espirituales, vitales, estéticos, etcétera, deberían integrarse en la educación en valores.

c. Dejar de creer que la educación en valores solo es un trabajo de aula, aislado de las relaciones e instituciones sociales. El aula aporta los elementos indispensables, pero que deben ponerse a prueba en las relaciones familiares, amicales y sociales, en general, y también en las instituciones que la afirman o impiden su realización. Es en ese entramado donde los valores (y la vida ética) miden su real impacto.

Finalmente, si bien es cierto que es importante el sentido antropológico de la educación en valores, no puede perderse de vista el contexto de esa realización. Somos seres 
en el mundo que creamos y recreamos mundo. ¿En qué y para qué mundo? La pandemia nos ha mostrado los graves problemas que tenemos como humanidad, por lo que son los retos globales (deterioro del medioambiente, pobreza, nuevas pandemias, justicia internacional, riesgo de desaparición de la especie humana, etcétera) los que deberían estar integrados en la educación con valores. Sin embargo, esta tarea titánica debe tener un principio integrador. Y ese principio tendrá que pasar por el ejercicio del pensamiento, la voluntad y las emociones de las personas.

\section{REFERENCIAS}

Carreras, Ll., Eijo, P., Estany, A., Gómez, M. T., Guich, R., Mir, V., Ojeda, F., Planas, T., y Serrats, M. G. (1996). Cómo educar en valores. Materiales, textos, recursos y técnicas. Narcea.

Díaz, C. (2001). Las claves de los valores. EIUNSA.

Ferreiros, P. (1983). Los valores en la educación. En M. Bartolomé, P. Ferreiros, J. Fondevila y M. Morilla, Educación y valores. Sobre el sentido de la acción educativa en nuestro tiempo (pp. 203-234). Narcea.

Gracia, D. (2000). Fundamentación y enseñanza de la bioética. El Búho.

Kohlberg, L. (1992). Psicología del desarrollo moral. Desclée de Brouwer.

Maslow, A. (1991). Una teoría de la metamotivación: las raíces biológicas de la vida valorativa. En R. Walsh y F. Vaughan (Comp.), Más allá del ego. Textos de psicología transpersonal (pp. 182-196). Kairós.

Mencio. (2012). Mencio. Ediciones en Lenguas Extranjeras.

Morin, E. (1999). Los siete saberes necesarios para la educación del futuro. Unesco.

Paniego, J. A. (1999). Cómo podemos educar en valores. Editorial CCS.

Payá, M. (2000). Educación en valores para una sociedad abierta y plural: aproximación conceptual. Desclée de Brouwer.

Polo, M. Á. (2005). El significado de educar en valores. Reflexión y Crítica. Revista de Filosofía, 9(5), 100-110.

Polo, M. Á. (2008). Los problemas en los discursos de los valores. Reflexión y Crítica. Revista de Filosofía, 12(6), 54-64. 